\title{
A Complete Approach for delimitation of Data in Cloud Environment
}

\author{
Chen Ming1), Debnath Bhattacharyya ${ }^{2)}$
}

\begin{abstract}
In the past, the focus of the computer is cloud computing, large data and Internet of things (IOT). No matter what computer you use, you always need to store it. The challenge in this regard is to store large amounts of data and their integrity. In the cloud, computing resources and services are available on demand, the most important of which is data storage. In this paper, the focus is on the study of the literature by providing a building and a comprehensive overview of the different deduplication technologies used in distributed storage. Deduplication (de-duplication) technology is increasingly being accepted as an effective technology to reduce storage costs, where deduplication technology has become an important part of storage infrastructure. This paper also reviews the backdrop and explanation features of information deduplication, and systematizes the technical research of digital repeat data repetition techniques, presenting a dynamic process flow. The summary and cataloging of high-resolution repetitive data helps to provide up-to-date plan deliberation for information deduplication. Finally, we summarize the open issues and future explorations in deduplication based on storage systems.
\end{abstract}

Keywords : Deduplication, Data, Access control, Proxy re-encryption, Memory management, Data deduplication

\section{Introduction}

Distributed computing offers another method for Information Technology benefits by revising different assets (e.g., capacity, figuring) and giving them to clients in view of their requests. Distributed computing gives a major asset pool by connecting system assets together. It has alluring properties, for example, adaptability, flexibility, adaptation to non-critical failure, and

\footnotetext{
Received(May 1, 2018), Review Result(1st: May 17, 2018, 2nd: June 15, 2018), Accepted(June 22, 2018)

${ }^{1}$ Harbin University of Commerce. Engineer in the Computer Science Experimental Center of Harbin University of Commerce email: chenming4321@126.com

${ }^{2}$ (Corresponding Author)Department of Computer Science and Engineering, Vignan's Institute of Information Technology, Visakhapatnam, AP, India

email: debnathb@gmail.com
} 
pay-examine. In this manner, it has turned into a promising administration stage. The most critical and prevalent cloud benefit is information stockpiling administration. Cloud clients transfer individual or private information to the server farm of a Cloud Service Provider (CSP) and enable it to keep up this information. Since interruptions and assaults towards touchy information at CSP are not avoidable[1], it is judicious to expect that CSP can't be completely trusted by cloud clients. In addition, the loss of control over their very own information prompts high information security dangers, particularly information protection spillages[2].

Because of the quick advancement of information mining and different investigation innovations, the protection issue ends up plainly genuine[2]. Consequently, a great practice is to just outsource scrambled information to the cloud to guarantee information security and client protection. Be that as it may, the same or distinctive clients may transfer copied information in encoded frame to CSP, particularly for situations where information is shared among numerous clients. In spite of the fact that distributed storage space is gigantic, information duplication significantly squander organize assets, devour a great deal of vitality and confounds information administration. The advancement of various administrations additionally makes it earnest to convey effective asset administration systems. Thus, deduplication ends up plainly basic for huge information stockpiling and handling in the cloud. Deduplication has demonstrated to accomplish high cost reserve funds[3-5].

Clearly, the funds, which can be passed back specifically or in a roundabout way to cloud clients, are noteworthy to the financial aspects of cloud business. The most effective method to oversee scrambled information stockpiling with deduplication in a productive way is a handy issue. Be that as it may, current modern deduplication arrangements can't deal with scrambled information. Existing answers for deduplication experience the ill effects of savage power assaults[6][7]. They can't adaptably bolster information get to control and denial at the same time[8]. Most existing arrangements can't guarantee unwavering quality, security and protection with sound execution. Practically speaking, it is difficult to enable information holders to oversee deduplication because of various reasons. To begin with, information holders may not be constantly on the web or accessible for such an administration, which could cause stockpiling delay. Second, it might interfere the protection of information holders during the time spent finding copied information. Forward, an information holder may have no clue how to issue information get to rights or deduplication keys to a client in a few circumstances when it doesn't know other information holders because of information super-circulation. 


\section{Background Work}

Z. Yan[9], gave intermediary re-encryption and possession test to deduplicate scrambled information put away in cloud. The fundamental favorable position of these procedures is that clients can share information notwithstanding when they are disconnected. The fundamental disservice of these strategies is that enhancement of configuration is important with the goal that CSP capacities legitimately in deduplication administration.

M. Bellare[1], gave DupLESS that gives secure reduplicated stockpiling to oppose savage power attacks. In DupLESS, a gathering of associated customers (e.g., organization representatives) scramble their information with the guide of a Key Server (KS). The fundamental preferred standpoint of these systems is that animal power assaults are maintained a strategic distance from and customers can encode their information with key server which is not quite the same as isolated stockpiling server. The principle disadvantage of these systems is that adaptability to other information clients cannot be given.

C. Y. Liu[10], an arrangement based deduplication intermediary conspire was proposed however it didn't consider copied information administration (e.g., cancellation and proprietor administration) and did not assess plot execution. The principle favorable position of this method is that it sets up trust connection among distributed storage parts with strategy based deduplication. The fundamental inconvenience of this system is that information erasure and proprietor administration isn't considered by arrangement based deduplication.

T. Y. Wu[11], proposed Index Name Servers (INS) to oversee not just document stockpiling, information deduplication, advanced hub determination, and server stack adjusting, yet in addition record pressure, lump coordinating, constant input control, IP data, and occupied level list checking .The principle favorable position of this method is that Index Name Servers calculation help to diminish workloads of assets and enhance the execution of system.INS likewise handles server stack balancing. - The fundamental impediment of this strategy is that encoded information can't be deduplicated. C. Fan, have given the framework in light of the supposition that CSP knows the encryption key of data. Thus it can't be utilized as a part of the circumstance that the CSP can't be completely trusted by the information holders or proprietors. The fundamental favorable position of these systems is that it bolsters deduplication on plaintext and ciphertext. The principle weakness of this procedure is that it doesn't bolster encoded information deduplication. 


\section{Data Deduplication Challenges}

Data deduplication is a system used for diminishing the measure of storage space that an affiliation requires to save. In various affiliations, the limit structures contain duplicate copies of various data. Consider the situation where a comparative record may be saved in a couple of better places by different customers, or possibly two archives that aren't indistinct may at show consolidate a noteworthy piece of comparative data. Deduplication executes the additional extra replica by saving just a single copy of the data and supplanting interchange copies with pointers so as to the principal copy. Affiliation a significant part of the time use deduplication in support and calamity recovery applications and in fundamental accumulating that leads profitable limit organization. In clear, deduplication occurs on the record level; that is, it executes duplicate copies of a comparative archive. This kind of deduplication is known as record level deduplication or single event storing (SIS).

Square level deduplication arranges for more space than SIS, and a specific sort known as factor piece or variable length deduplication has turned out to be generally well known. Regularly the expression "information deduplication" is utilized as square level/variable length deduplication. The information deduplication can lessen the measure of plate or tape that the association is in requires, and thus diminishes costs. NetApp reports says that there is distinctive cases in which deduplication can lessen capacity prerequisites up to 95 percent, and the sort of information that you're attempting to deduplicate and the measure of record sharing your association wills impact your own deduplication proportion. While deduplication can be connected to information put away on circle, the moderately much expenses of plate stockpiling make deduplication an exceptionally prevalent choice for circle based frameworks.

\section{Proposed System}

A realistic arrangement to manage the encoded colossal data in the cloud with deduplication in light of ownership test and Proxy re-encryption has been proposed. This procedure is used for versatile data updates and conferring to de-duplication despite when the data holders are disengaged. Mixed information can be steadily gotten to in light of the way that solitary endorsed information possessors can get the similar keys utilized for data unscrambling. 


\section{Proposed System Process Modules}

\subsection{Encrypted Data Upload:}

In the event that information duplication check is negative, the information holder scrambles its information utilizing an arbitrarily chose symmetric key DEK keeping in mind the end goal to guarantee the security and protection of information, and stores the encoded information at CSP together with the token utilized for information duplication check.

\subsection{Information Deletion:}

At the point when the information holder erases information from CSP, CSP initially deals with the records of copied information holders by expelling the duplication record of this client. In the event that the rest records are not vacant, the CSP won't erase the put away encoded information, however square information access from the holder that solicitations information cancellation. In the event that the rest records are vacant, the scrambled information ought to be expelled at CSP.

\subsection{Information Owner Management:}

In the event that that a genuine information proprietor transfers the information later than the information holder, the CSP can figure out how to spare the information scrambled by the genuine information proprietor at the cloud with the proprietor created DEK and later on, AP underpins re-encryption of DEK at CSP for qualified information holders.

\section{System Architecture}

In the proposed investigate work to design and execute a system which will give the parallel dealing with to recognize the data de-duplication issue in conveyed capacity. We overhaul our system in security. Specifically, we display a moved arrangement to help more grounded security by scrambling the record with differential advantage keys. Thusly, the customers without looking at benefits can't play out the duplicate check. Also, such unapproved customers can't translate the figure message even plan with the S-CSP. Safety examination make obvious that the current system is safe and the extent that the explanation decided in the planned safety show.

As appeared in Fig. 1, the framework includes three sorts of substances: 1) CSP that offers 
stockpiling administrations and can't be completely convicted since it is interested about the substance of put away information, however ought to perform genuinely on information stockpiling so as to increase business benefits; 2) information holder (ui ) that transfers and spares its information at CSP. In the framework, it is conceivable to have various qualified information that might spare the similar encoded crude information in CSP. The information owner that produces or makes the record is viewed as information proprietor. It has higher need than other typical information holders, which will be displayed in Section 4; 3) an approved gathering (AP) that does not intrigue with CSP and is completely convicted by the information holders to confirm information proprietorship and knob information deduplication. For this situation, AP can't know the information put away in CSP and CSP ought not to know the plain client information in its stockpiling. Proposed plot contain following fundamental viewpoints,

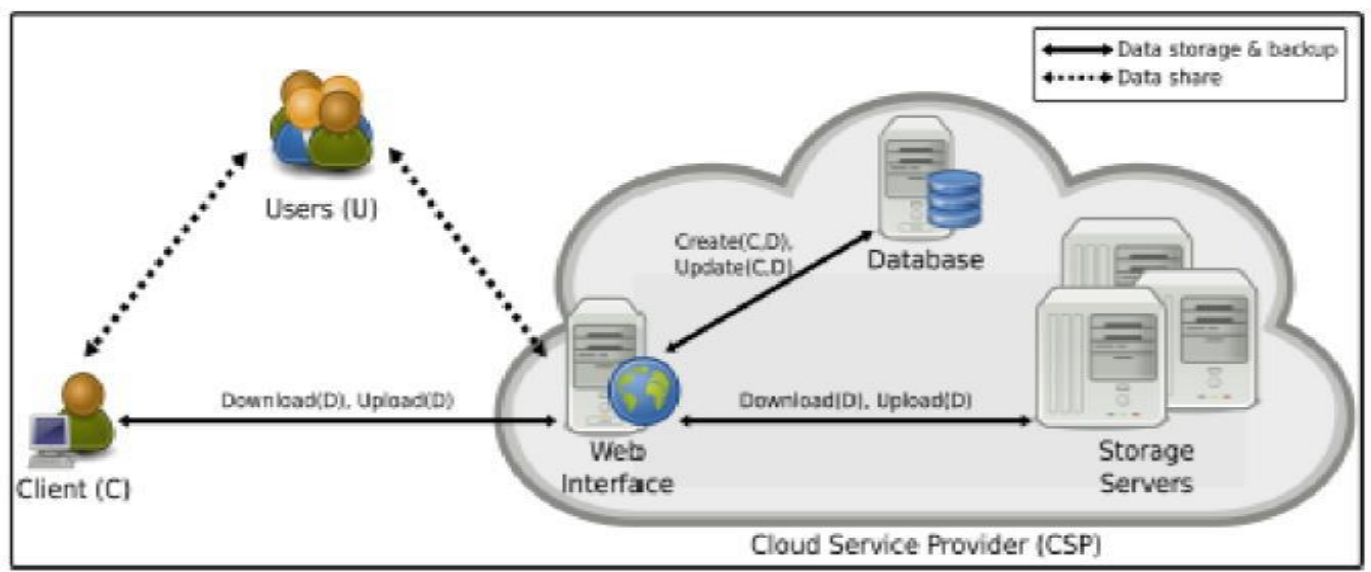

[Fig. 1] The architecture of the model

\subsection{Proposed Algorithm}

In 1978, Ron Rivest, Adi Shamir, and Leonard Adleman exhibited a new technique for count, which was fundamentally to supplant the fewer safe National Bureau of Standards (NBS) algorithm.RSA figuring is lopsided cryptography computation. Disproportionate truly suggests that it wears down two various keys i.e. Open Key and Confidential Key. As the name depicts that the Community Key is given to everyone and Confidential Key is kept Confidential.

\section{A case of unbalanced cryptography:}


1. A customer (for instance program) sends its open key to the server and solicitations for a few information.

2. The server encodes the information utilizing customer's open key and sends the scrambled information.

3. Client gets this information and decodes it.

\section{Performance Analysis}

Joined encryption experiences a few shortcomings which have been generally talked about in the writing, As the encryption key relies upon the estimation of the plaintext, an aggressor who has accessed the capacity can execute the alleged "word reference assaults" by contrasting the ciphertexts coming about because of the encryption of understood plaintext esteems from a lexicon with the put away figure writings. To be sure, regardless of whether encryption keys are scrambled with clients' private keys and put away elsewhere, the conceivably malevolent cloud supplier, who has no entrance to the encryption key however approaches the encoded lumps (pieces), can without much of a stretch perform disconnected word reference assaults and find unsurprising documents. This issue emerges in where lumps are put away at the capacity supplier in the wake of being scrambled with focalized encryption. To be sure, regardless of whether encryption keys are scrambled with clients' private keys and put away elsewhere, the conceivably malevolent cloud supplier, who has no entrance to the encryption key however approaches the encoded lumps (pieces), can without much of a stretch perform disconnected word reference assaults and find unsurprising documents. Since this has guide access to server information are less secure. In property based encryption the multifaceted nature of usage is high and it isn't adaptable and adaptable for substantial volume of information however this backings vast number of copy duplicates so it is much appropriate for little information that has much duplicates.

Since it is executed in symmetric key strategy the key dissemination and calculation is the more concerning issue and could harm when packed. The information that should be put away is first preprocessed if vital. In specific cases the preprocessing takes up a great deal of time in light of the kind of preparing that is actualized. Information cleaning, Normalization, Data concealing, Structuring of information, and so forth are a portion of the preprocessing steps accessible. The following stage is the lumping. The way toward part the given information into any squares or pieces of information is called as lumping. This is the pivotal advance in the deduplication procedure. This is on account of, in light of the extent of each piece the quantity 
of copy information changes. The information that should be put away is first preprocessed if vital. In specific cases the preprocessing takes up a great deal of time in light of the kind of preparing that is actualized. Information cleaning, Normalization, Data concealing, Structuring of information, and so forth are a portion of the preprocessing steps accessible. In light of the underlying information, the lump size ought to be settled such that they got pieces have extensive number of copies and in this way the capacity size will be lessened however much as could reasonably be expected.

\section{Results of Deduplication}

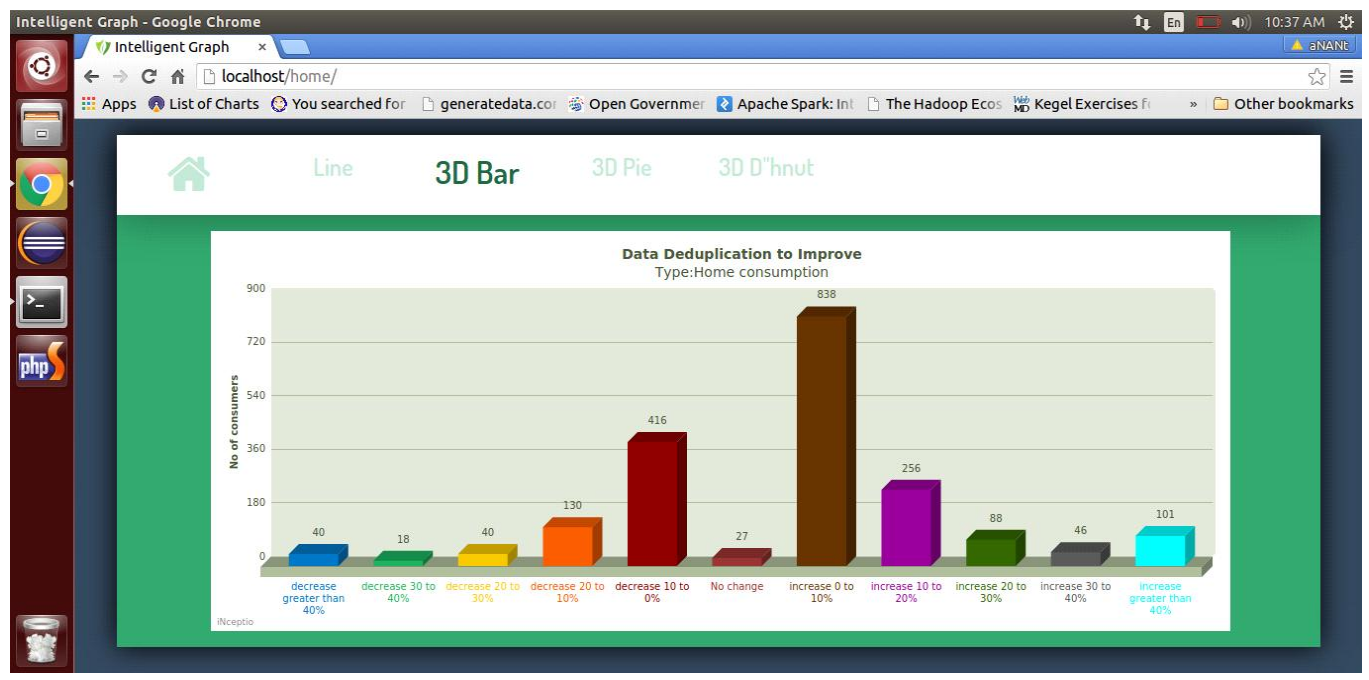

[Fig. 2] Duplication representation in terms of Bar Graph 


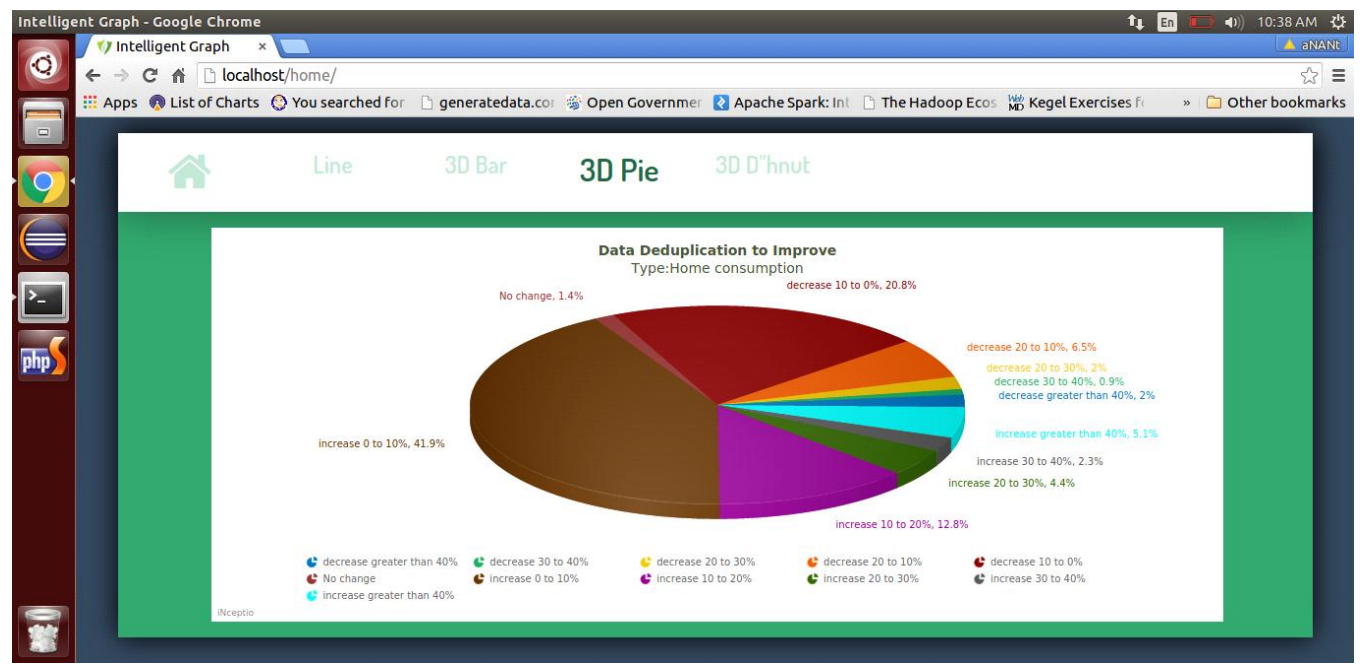

[Fig. 3] Duplication representation in terms of Pie Diagram

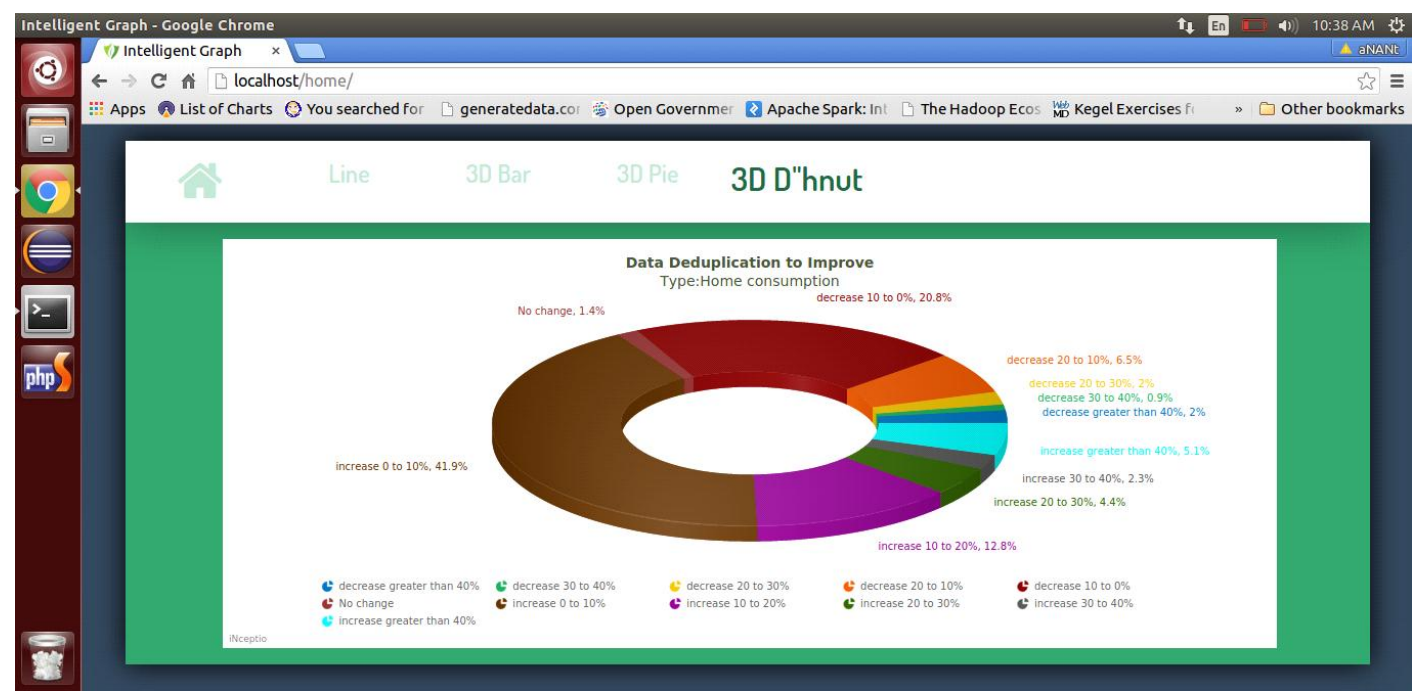

[Fig. 4] Duplication representation in terms of 3D Diagram

\section{Conclusion and Future Work}

A new trend has set in storage where data de-duplication plays, a novel role in compression and other data reduction in high-tech element of the solution. Data deduplication technique get better information defense, which amplify the velocity of repair, and decreases the expenses and the use of bandwidth. De-duplication is integrated with cloud storage space, this helps in the 
easy maintenance of data and eradicating the replica's in the cloud server. Cloud computing, storage resources is efficiently utilized this allows every organization to build their own private cloud and hybrid cloud according to their purposes and needs. To meet the rising performance need, improvement is developed to meet the growing need for SSSD(System Security Services Daemon) gives an arrangement of daemons to oversee access to remote catalogs and validation systems.

\section{References}

[1] M. Bellare, S. Keelveedhi, and T. Ristenpart, DupLESS: Server-Aided Encryption for Deduplicated Storage. Proceedings of the 22nd USENIX Conference on Security, (2013) August 14-16; Washington, USA, pp.179-194.

[2] J. Hur, D. Koo, Y. Shin, and K. Kang, Secure Data Deduplication with Dynamic Ownership Management in Cloud Storage, IEEE Transaction on Knowledge and Data Engineering (2016), Vol.28, No.11, pp.3113-3125.

[3] S. R. Deepu, R. Bhaskar, and B. S. Shylaja, Performance Comparison Of Deduplication Techniques For Storage In Cloud Computing Environment, Asian Journal of Computer Science Information Technology (2014), Vol.4, No.5, pp.42-46.

[4] Y. Zhang, D. Feng, H. Jiang, W. Xia, M. Fu, F. Huang, and Y. Zhou, A Fast Asymmetric Extremum Content Defined Chunking Algorithm for Data Deduplication in Backup Storage Systems, IEEE Transactions on Computers (2016), Vol.66, No.2, pp.199-211.

[5] X. Du, W. Hu, Q. Wang, and F. Wang, ProSy: A similarity based inline deduplication system for primary storage. Proceedings of IEEE International Conference on Networking, Architecture and Storage(NAS), (2015) August 6-7; Massachusetts, USA, pp.195-204.

[6] B. Mao, H. Jiang, S. Wu, and L. Tian, Leveraging Data Deduplication to Improve the Performance of Primary Storage Systems in the Cloud, IEEE Transactions on Computers (2016), Vol.65, No.6, pp.1775-1788.

[7] S. Luo, G. Zhang, C. Wu, S. Khan, and K. Li, Boafft: Distributed Deduplication for Big Data Storage in the Cloud, IEEE Transactions on Cloud Computing (2015), Vol.61, No.11, pp.1-13.

[8] W. Xia, H. Jiang, D. Feng, and L. Tian, DARE: A Deduplication-Aware Resemblance Detection and Elimination Scheme for Data Reduction with Low Overheads, IEEE Transactions on Computers (2016), Vol.65, No.6, pp.1692-1705.

[9] Z. Yan, W. Ding, X. Yu, H. Zhu, and R. H. Deng, Deduplication on Encrypted Big Data in Cloud, IEEE Transactions on Big Data (2016), Vol.2, No.2, pp.138-150.

[10] C. Y. Liu, X. Liu, and L. Wan, Policy-Based De-depulication in Secure Cloud Storage. Proceedings of International Conference on Trustworthy Computing and Services, Communications in Computer and Information Science (2013), Vol.320, pp.250-262. 
[11] T. Y. Wu, J. S. Pan, and C. F. Lin, Improving Accessing Efficiency of Cloud Storage Using De-Duplication and Feedback Schemes, IEEE Systems Journal (2014), Vol.8, No.1, pp.208-218. 